Proceedings of the XXXVIII International School and Conference on the Physics of Semiconductors "Jaszowiec" 2009

\title{
Rocking Curve Imaging Studies of Laterally Overgrown GaAs and GaSb Epitaxial Layers
}

\author{
A. WierzBickA ${ }^{a * *}$, D. LÜBbert ${ }^{b}$, J.Z. DomagaŁA ${ }^{a}$ And Z.R. ZytKieWiCZ ${ }^{a}$ \\ ${ }^{a}$ Institute of Physics, Polish Academy of Sciences, al. Lotników 32/46, 02-668 Warszawa, Poland \\ ${ }^{b}$ Humboldt-Universität, Newtonstr. 15, 12489 Berlin, Germany
}

\begin{abstract}
X-ray rocking curve imaging technique was used to study crystallographic perfection of laterally overgrown epitaxial structures. We focus on rocking curve imaging studies of Si-doped GaAs and GaSb laterally overgrown layers grown by liquid phase epitaxy on $\mathrm{SiO}_{2}$ masked GaAs and GaSb/GaAs substrates, respectively. High spatial resolution offered by rocking curve imaging technique allows studying the effect of laterally overgrown epitaxial wing tilt towards the mask. Distribution of tilt magnitude over the area of laterally overgrown epitaxial stripes is easily determined. In heteroepitaxial $\mathrm{GaSb} / \mathrm{GaAs}$ laterally overgrown epitaxial structures local mosaicity in the wing area was detected. Since individual grains are clearly visible on rocking curve imaging maps, their size and relative misorientation can be determined.
\end{abstract}

PACS numbers: 61.05.cp, 68.55.ag, 81.15.Lm

\section{Introduction}

Modern semiconductor electronics requires fast techniques that allow precise analysis of structural quality of crystalline materials, starting from bare wafers up to complicated semiconductor structures. One of techniques developed recently is synchrotron radiation based rocking curve imaging (RCI). In this work the RCI was applied for detection and visualization of crystalline lattice microdefects in laterally overgrown epitaxial (ELO) layers. Since ELO structures consist of a few hundred micrometers wide monocrystalline stripes regularly arranged on a template (see Fig. 1) they are seen by an X-ray beam as a periodic distribution of strain and defects. Thus, ELO layers are perfectly suitable to demonstrate a potential of the RCI technique.

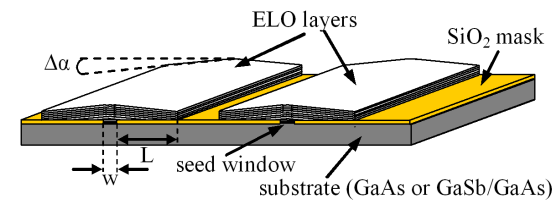

Fig. 1. Schematic drawing of ELO structure. $L$ and $w$ denote widths of the ELO wing and of the seeding line, respectively. $\Delta \alpha$ is the maximal tilt angle of the ELO lattice planes.

\section{Experimental}

Rocking curve imaging is a technique which combines the features of X-ray imaging (very high spatial resolu- tion) and X-ray diffractometry (very high angular resolution). Experiments reported here were performed at the beamline ID 19 of the ESRF, Grenoble, France in the following way (see Fig. 2): a wide, homogeneous and monochromatic synchrotron X-ray beam illuminated a large part of the sample. A precise goniometer allowed rotating the sample close to the Bragg position with very small angular step around the axis perpendicular to the diffraction plane. For each angular position of the sample series of local diffraction images were acquired by using very fast detector (Frelon camera $-2048 \times 2048$ pixels, pixel size equals to $1.4 \mu \mathrm{m})$. Then, all digital images collected were used to create RCI maps of the sample that later on were visualized and analyzed with dedicated software [1]. The samples studied in this work were Si-doped GaAs and GaSb layers grown by liquid phase epitaxy on $\mathrm{SiO}_{2}$-masked GaAs and GaSb/GaAs substrates, respectively. More details of the growth procedure can be found elsewhere [2].

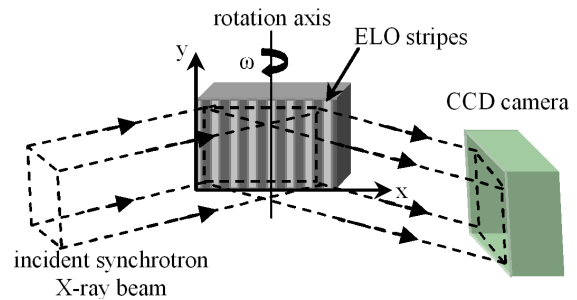

Fig. 2. Schematic drawing of RCI setup. A wide beam of monochromatic X-rays is diffracted on ELO sample. Diffraction plane is oriented horizontally, perpendicular to ELO stripes.

* corresponding author; e-mail: czyzak@ifpan.edu.pl 


\section{Results and discussion}

First we focus on the RCI analysis of a single GaAs ELO stripe. Figure 3a shows spatial distribution of the Bragg peak position over wide area of the sample. Signals coming from the substrate, ELO wings and the material grown vertically over the window in the mask are easily distinguishable. Let us note that the Bragg angle continuously increases from the left to the right edge of the ELO stripe. This is an indication of downward wing tilt - the phenomenon commonly observed in many ELO systems and being due to an interaction of laterally overgrown parts of the layer with the mask underneath [3]. The value of the wing tilt angle $\Delta \alpha \approx 0.3^{\circ}$ can be easily determined from Fig. 3a. Moreover, a high uniformity of wing tilt along the ELO stripe is clearly visible. As will be shown later, this is not necessarily the case in heteroepitaxial ELO layers where lattice and thermal expansion mismatches lead to strongly stressed epilayers. Figure $3 \mathrm{~b}$ shows spatial distribution of the full width at half maximum (FWHM) of local X-ray rocking curves measured from the same piece of ELO stripe. As seen, FWHM is the largest in the central part of the layer. Figure 4 shows a cross-section at the position $y=450$ of the 3-dimensional map from Fig. 3a. This is more precise way of showing how the Bragg peak position changes across the ELO stripe. In particular, Fig. 4 shows that the fastest changes of the Bragg angle take place in the central part of the layer. Thus, the crystal lattice there must be strongly strained, which explains enhanced values of FWHM in that part of the layer (cf. Fig. 3b).

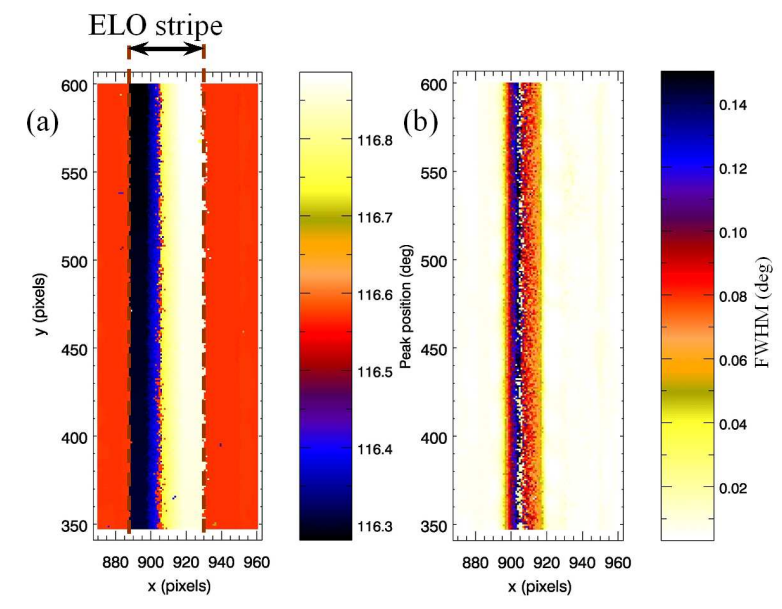

Fig. 3. Spatial distribution of Bragg peak position (a) and map of local rocking curve FWHM values (b) in GaAs/GaAs single ELO stripe. Pixel size is $1.4 \mu \mathrm{m}$.

Having clarified behavior of homoepitaxial structures we have used the RCI technique to analyze crystallographic quality of heteroepitaxial ELO layers in which lattice parameter and thermal expansion mismatch with the substrate are additional sources of strain. As an example, Fig. 5 shows RC map of GaSb ELO stripe grown on GaAs substrate coated by a $2 \mu \mathrm{m}$ thick planar GaSb

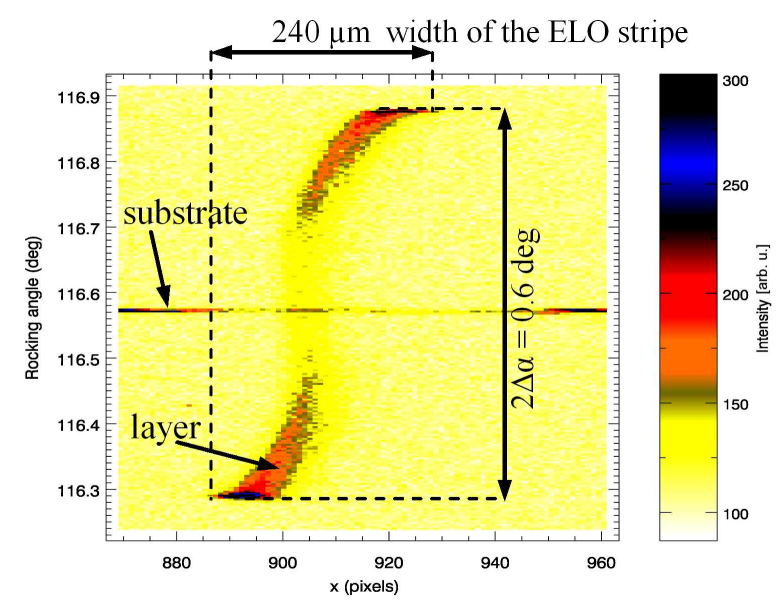

Fig. 4. RC map of the GaAs/GaAs single ELO stripe.

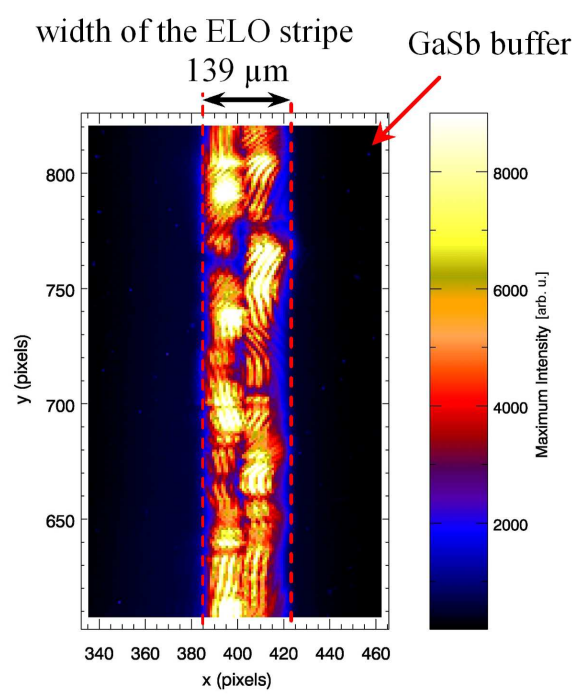

Fig. 5. Spatial distribution of local diffraction intensity in GaSb/GaAs ELO layer. Pixel size is $1.4 \mu \mathrm{m}$. Microblock structure of the layer is visible.

buffer and $\mathrm{SiO}_{2}$ mask. As before, signals originating from the buffer, ELO wings and seeding window are clearly distinguished. This time, however, the GaSb ELO layer diffracts X-rays much stronger than the substrate (GaSb buffer), which is a direct proof that crystal lattice quality improves during ELO growth procedure. In addition, distribution of diffracted signal intensity over the stripe area is inhomogeneous and some texture is visible. Due to a very high spatial resolution of the RCI technique individual GaSb grains (microblocks) are visualized. Thus, their average size, being in the range of $\approx 110-150 \mu \mathrm{m}$, can be readily measured. From RC maps presenting distribution of the Bragg peak position (not shown here) wing tilt angle of $\approx 250$ arcsec can be found for the sample studied. Moreover, relative misorientation of neighboring GaSb microblocks has been measured to be $\approx 40$ arcsec. 


\section{Conclusions}

Rocking curve imaging technique was successfully used to analyze crystallographic quality of laterally overgrown epitaxial structures. Wing tilt towards the mask was observed and homogeneity of the tilt magnitude over the sample area was observed for homoepitaxial GaAs/GaAs ELO structure. Presence of microblock structure in GaSb/GaAs ELO layer was detected. Due to intense synchrotron X-ray beam and application of an efficient detector that led to very high spatial resolution of the RCI technique, individual GaSb microblocks were imaged. Their misorientation relative to the GaSb buffer was measured.

\section{Acknowledgments}

This work was supported by the ESRF/MA/623/2009 project of the Polish Ministry of Science and Higher Education and by the European Union within European Regional Development Fund, through grant Innovative Economy (POIG.01.01.02-00-008/08).

\section{References}

[1] D. Lübbert, T. Baumbach, J. Appl. Cryst. 40, 595 (2007).

[2] Z.R. Zytkiewicz, Cryst. Res. Technol. 34, 573 (1999).

[3] Z.R. Zytkiewicz, Thin Solid Films 412, 64 (2002). 